Muro de la Investigación, 2019(2), agosto-diciembre ISSN: 2523-2886

DOI: https://doi.org/10.17162/r-muro-investigaion.v4i2.842

\title{
COMPETENCIAS ORGANIZACIONALES Y DESEMPEÑO LABORAL EN LOS LÍDERES DE UNA IGLESIA CRISTIANA BRASILERA
}

\author{
Organizational skills and work performance in the leaders of the christian Church in \\ Brazil
}

Andrea Possato Derevetski Trindade ${ }^{1}$

Universidad Peruana Unión, Perú ${ }^{1}$

Recibido: 30 marzo de 2019

Aceptado: 01 de julio de 2019

\begin{abstract}
Resumen
Se parte de la premisa que, en los últimos años, el entorno de relacionamiento de las organizaciones se ha tornado altamente competitivo. Esto se fundamenta en la existencia de grandes identidades que generan un empoderamiento definitorio en sus contextos; donde es necesario considerar que las bases estructurales de las empresas se hallan en su talento humano como eje de producción. En función de las competencias de cada uno y su desempeño laboral, dichas bases se ven estrechamente ligadas al contexto de desarrollo en materia de autorrealización, confort o superación. Ello también es aplicable al contexto institucional de la Iglesia Adventista Séptimo Día (IASD), caracterizada por promover principios de la cultura cristiana protestante desde su incepción en el año 1863; a partir del cual se ha expandido con miembros y socios a nivel mundial. Esto le ha permitido constituir una identidad sólida referente a sus procesos a lo largo de su existencia. Por ello, el presente trabajo de investigación surgió con el objetivo de analizar la relación existente entre las variables de competencia organizacional y el desempeño laboral en los líderes de la IASD en la ciudad de Campinas, una totalidad muestral de 71 pastores, a quienes se les aplicó una encuesta propuesta por 13 dimensiones, fundamentadas en las variables de acción de toda organización y su repercusión en los resultados reales de las mismas. Cabe acotar que se halló un valor adecuado entre las competencias organizacionales y el desempaño laboral de los empleados, siendo esta última variable dependiente de la primera, lo que influye de manera directa en las realidades propias de cada empresa o institución bien consolidada a nivel global.
\end{abstract}

Palabras Clave: Competencias Organizacionales, Desempeño Laboral, Competitividad, Cultura Cristiana, Empleados 


\begin{abstract}
This paper is based on the premise that in the environment of relationships of organizations has become highly competitive in recent years, based on the existence of large identities that generate a defining empowerment in their contexts, where it is necessary to consider that the structural basis of companies are in their human talent as a production axis, where depending on the competences of each one and their work performance are closely linked to the context of their development, in terms of self-realization, comfort or improvement. This is applicable to the institutional context of the Seventh-day Adventist Church (IASD, in Spanish), characterized by promoting principles of the Christian Protestant culture, emerging in the year 1863, from which it has expanded with members and partners worldwide, which has allowed it to constitute a solid identity regarding its processes with more than fourteen decades in progress. Therefore, this research work arose with the objective of analyzing the relationship between the variables of organizational competence and work performance in the leaders of the IASD in the city of Campinas, a sample total of 71 pastors, who are applied a survey proposed by 13 dimensions, based on the variables of action of any organization and its impact on their actual results. It should be noted that an adequate value was found between the organizational competences and the labor performance of the employees, the latter being dependent on the first variable, which directly influences the realities of each well-established company or institution globally.
\end{abstract}

Key Words: Organizational Competencies, Labor Performance, Competitiveness, Christian Culture, Employees

\title{
Introducción
}

Dentro de la cultura organizacional, existen múltiples connotaciones que se derivan de las variables actitudinales de la empresa en materia de gestión de su talento humano, considerado como cimiento de sus procesos productivos y aspecto elemental de su éxito organizacional. Por ello, en los últimos años, se ha otorgado una amplia importancia al liderazgo como fenómeno de movilización de las masas y de integración en el contexto empresarial, donde se establece una estrecha interrelación entre la consecución del ambiente de desarrollo del talento humano y el desempeño laboral. Estos se derivan de las condiciones de mejoramiento de los índices de calidad de vida de los que les provea la organización de la cual dependen.

En este sentido, el estudio de la relación entre estos aspectos despierta un amplio interés dentro del contexto académico, a partir del carácter de movilización en las masas que surge de los fenómenos socioeconómicos potencializados en una sociedad posmoderna. Este es el motivo por el cual surge el presente proyecto de investigación de carácter cuantitativo, orientado a generar un análisis del contexto actual del comportamiento y la relación hallada 
entre las competencias organizacionales y el desempeño laboral de los líderes de la Iglesia Adventista Séptimo Día (IASD) en la ciudad brasilera de Campinas, en el año 2016. Este trabajo tiene el propósito de establecer un aporte en cuanto a la importancia de la consecución adecuada ese estos parámetros de desarrollo organizacional y sus repercusiones en una entidad como la IASD, gozadora de más de un siglo de funcionamiento y prestigio en el globo.

\section{Marco Teórico}

Competencias organizacionales

En palabras de Camejo (2008), dentro de la cultura de las organizaciones, existe un conjunto de competencias derivadas de la necesidad de establecer políticas de acción que se traduzcan en el mejoramiento de la situación financiera de la empresa. Esto implica sus distintos públicos concernientes (stakeholders) y las repercusiones de sus procesos en los mismos, y viceversa. En este orden de ideas, es necesario resaltar que las competencias no se fundamentan en una serie de procesos regulados, sino que presentan una dimensión un tanto más compleja.

Alternativamente, Barrios y Fong (2002) señalan que las competencias organizacionales se constituyen como las capacidades mínimas necesarias para que un individuo o institución se desempeñe de manera adecuada en un contexto de acción determinado; a partir de principios de efectividad de procesos que se devienen por su constante profundización. Las mismas, son constituidas a partir de conceptos actitudinales predefinidos.

Dichas competencias se conciben a partir de la sumatoria de una serie de variables que encaminan la cultura de una organización, que a su vez se derivan en tres tipologías fundamentales: "saber," con base en las capacidades formativas; "hacer," en consideración de la experiencia ganada; y “ser," que va un poco más de la mano con los aspectos definitorios de cada individuo (Bohlander \& Sherman, 2011).

En este orden de ideas, Merten (2000) afirma que existen una serie de dimensiones derivadas de las competencias organizacionales, las cuales son: liderazgo, motivación, trabajo en equipo, comunicación, participación y estructura organizacional.

Desempeño laboral 
El desempeño laboral es comprendido como un fenómeno de carácter institucional que se deriva del conjunto de parámetros actitudinales manifiestos por los empleados a partir de sus condiciones motivacionales, en relación al nivel de exigencia que se predispone para cada tarea y el grado de remuneración y autosatisfacción que se deviene de su cumplimiento (Ivancevich, 2005).

Asimismo, es un frecuente objeto de estudio de investigadores en el ámbito organizacional, en tanto se determina que tiene implicaciones profundas en la realidad de la empresa. Con esto se aduce que los trabajadores son la base estructural de la misma, pues en dependencia de su nivel de implicación, se maximizaría la efectividad de los procesos colectivos y sería posible satisfacer en mayor medida las necesidades de los clientes objetivo (Aguirre, 2000).

Alternativamente, se halla la existencia de una serie de fases del desempeño laboral: 1) la identificación de parámetros actitudinales que se espera de la persona que ocupa un cargo determinado, estableciendo una relación entre lo que este es capaz de cubrir y los resultados ideales de acción; 2) la medición, donde se compara los resultados obtenidos con los esperados del desempeño de un trabajador en su campo de acción; 3) la gestión, fundamentada en la necesidad de establecer parámetros de mejoramiento de las actitudes de los trabajadores a partir de su constante capacitación para el alcance de mejores resultados de acción (Aguirre, 2000).

\section{Dimensiones del desempeño laboral}

El desempeño laboral está constituido a partir de una serie de variables que, evaluadas de forma individual y en conjunto, permiten conocer las discrepancias halladas en esta materia. Con ello, sería posible establecer estrategias de mejoramiento de la calidad de los procesos y de la prestación de servicios a los clientes, según lo amerite el caso (Universidad de Salamanca, 2004). Dichas dimensiones son:

- Puesto de trabajo, donde se constituyen los factores relacionados al ambiente en el cual se desenvuelve el trabajador en el cumplimiento de sus funciones.

- Línea de autoridad, donde se demarcan las responsabilidades de cada individuo, en materia de rendición de cuentas y de dependencia o supervisión de otros empleados de menor o mayor jerarquía 
- Ambiente de trabajo e integración, dimensión en la cual se toman en cuenta las redes de relacionamiento que conciernen al trabajador y su nivel de influencia, sea positivo o negativo.

- Comunicación y coordinación, en cuyo caso se considera la importancia de una comunicación interna sólida en la empresa como cimiento de la coordinación institucional.

- Condiciones ambientales, infraestructura y recursos, que caracterizan a la empresa como institución con una sede física, que en dependencia de cuyas condiciones el empleado podrá cumplimentar en mayor o menor medida sus roles de trabajo.

- Implicación en la mejora, fundamentado en la necesidad constante de cambio hallado en la empresa a partir de la inclusión de nuevos procesos, tecnologías y el fortalecimiento de los parámetros ya existentes.

- Motivación y reconocimiento, donde se toman en consideración parámetros ligados a la predisposición del trabajador por mejorar sus niveles de rendimiento en función a la autorrealización que se derive del cumplimiento de sus roles (Universidad de Salamanca, 2004).

\section{Dimensiones de las competencias organizacionales}

Liderazgo organizacional. Es un campo de trabajo que incluye el establecimiento de metas, tanto para individuos como para grupos de personas. Este campo de trabajo puede incluir elaborar grandes proyectos con empresas y corporaciones, e incluso proyectos más pequeños con grupos de personas que comparten puntos de vista similares. Las personas que buscan involucrarse en este campo deberán estar organizadas y orientadas a una visión. La capacidad de ver tanto la imagen pequeña como la grande es necesaria (Dávila, 2013).

Motivación. Esta es importante debido a su rol determinante en el rendimiento y a su naturaleza intangible. Desde el enfoque de recursos humanos, supone que las personas desean contribuir a la eficacia de la organización y pueden hacer contribuciones genuinas. La responsabilidad de la organización es crear un ambiente de trabajo que haga un uso completo de los recursos humanos disponibles (Dávila, 2013).

Trabajo en equipo. Se utiliza en gran cantidad de industrias con el fin de aumentar el rendimiento, la unidad de los empleados y la cultura de las empresas. Estas deben desarrollar frecuentemente nuevas ideas o productos utilizando un enfoque basado en proyectos reúnen equipos para difundir la responsabilidad (Dávila, 2013). 
Comunicación organizacional. Se enfoca principalmente en construir relaciones e interactuar con miembros internos de la organización y públicos externos interesados. Cómo se estructura una organización no solo es importante desde la perspectiva de la productividad y la eficiencia, sino principalmente cómo afecta la participación de quienes están empleados en esa organización (Dávila, 2013).

Estructura organizacional. Es un sistema que consiste en reglas y políticas institucionales explícitas e implícitas diseñadas para delinear la comisión, el control y la coordinacion de las diversas funciones y responsabilidades del trabajo. La estructura organizacional también determina cómo fluye la información de un nivel a otro dentro de la empresa. Una estructura organizacional exitosa define el trabajo de cada empleado y cómo encaja dentro del sistema general (Dávila, 2013).

\section{Metodología}

Diseño de investigación

El presente proyecto de investigación se constituyó a partir del establecimiento de una serie de parámetros metodológicos que permitieron cumplimentar su realización. En primer lugar, se empleó la metodología de carácter descriptivo (Monje, 2011), con base en la observación de las variables de objeto de estudio, sin alterar su comportamiento habitual de forma deliberada. En segundo lugar, se tomó en cuenta la investigación de tipo correlacional, a partir del establecimiento de una relación hallada entre las dos variables de estudio, generando un diagnóstico situacional adecuado a la temática predispuesta (Baptista, Fernández, \& Sampieri, 2014).

En otro orden de ideas, se concibió un diseño investigativo de carácter no experimental, en que se concibe la no manipulación deliberada de las variables de estudio, estipulando únicamente de forma analítica los fenómenos concernientes a su contexto habitual de comportamiento (Baptista, Fernández, \& Sampieri, 2014).

Asimismo, para la comprobación de hipótesis se utilizó el estadístico chi-cuadrado, que es una medida de divergencia entre la distribución de sus datos y una distribución esperada o hipotética de su elección, lo que permitió probar la independencia o determinar la asociación entre variables de criterio y predictora (Tinoco, 2008). 
Población y muestra

Por una parte, se determinó una población o universo compuesto por 1.382 pastores de la asociación local de iglesias del Estado de Brasil, frente a una muestra de 70 pastores pertenecientes a la IASD de la ciudad de Campinas, Sao Paulo, a partir del cual se estableció una serie de técnicas e instrumentos de recolección de datos pertinentes a la evaluación del objeto de estudio.

Técnicas e instrumentos de recolección de datos

Con base en la técnica de la encuesta, fue diseñado un cuestionario como instrumento de recolección de información, aplicado de forma específica a los pastores de la IASD, con el objetivo de obtener información cuantificable. Esto se estructuró en dos partes: desempeño laboral y competencias organizacionales. El primero comprende siete dimensiones, fundamentados en la escala de Likert como opciones de respuesta que facilitarían su posterior interpretación: 1) nunca, 2) casi nunca, 3) en ocasiones, 4) casi siempre y 5) siempre, respectivamente (Aristizábal, Ospina, \& Ramírez, 2003).

Técnicas de procesamiento y análisis de datos

Para la posterior gestión y análisis de los datos, se empleó el programa estadístico SPSS, el cual permitió no solo desplegar la información obtenida a partir de la realización de la encuesta en el campo de estudio, sino además evaluar la relación hallada entre las dos variables del instrumento diseñado, en función al chi-cuadrado. Esta metodología se estableció por Pearson, a fin de conocer la relación de las competencias organizacionales y el desempeño laboral de los líderes de la IASD que fueron entrevistados (Tinoco, 2008).

Sobre tales aspectos, se estableció una media ponderada en las respuestas, en función a su valor designado en la escala de Likert, para medir el nivel de satisfacción de forma generalizada y facilitar el establecimiento posterior de la relación entre las variables objeto de estudio (Aristizábal, Ospina, \& Ramírez, 2003).

\section{Resultados}


A continuación, se presentan de forma generalizada los resultados derivados de la aplicación e interpretación de la encuesta, desde sus dos sub-dimensiones concebidas para la posterior correlación:

Competencias Organizacionales

La aplicación de la encuesta permitió determinar una aproximación de la realidad que presentó la variable "competencia organizacional," para lo cual, fue necesario efectuar el análisis de cada dimensión, lo que presentó cada una de las preguntas aplicadas, partiendo de los resultados de la aplicación de la media ponderada:

Tabla 1

Resultados de la variable Competencias Organizacionales

\begin{tabular}{ccccc}
\hline $\mathrm{N}^{\circ}$ & Dimensión & $\begin{array}{c}\text { Media } \\
\text { ponderada }\end{array}$ & Porcentaje & Evaluación \\
\hline $\mathbf{1}$ & Liderazgo & 4,34 & $87 \%$ & Aceptable \\
$\mathbf{2}$ & Motivación & 4,64 & $93 \%$ & Satisfactorio \\
$\mathbf{3}$ & Trabajo en equipo & 4,04 & $80 \%$ & Aceptable \\
$\mathbf{4}$ & Comunicación & 3,95 & $79 \%$ & Regular \\
$\mathbf{5}$ & Participación & 3,92 & $78 \%$ & Regular \\
$\mathbf{6}$ & Estructura organizacional & 3,89 & $78 \%$ & Regular \\
Total & Promedio General & 4,13 & $83 \%$ & Aceptable \\
\hline
\end{tabular}

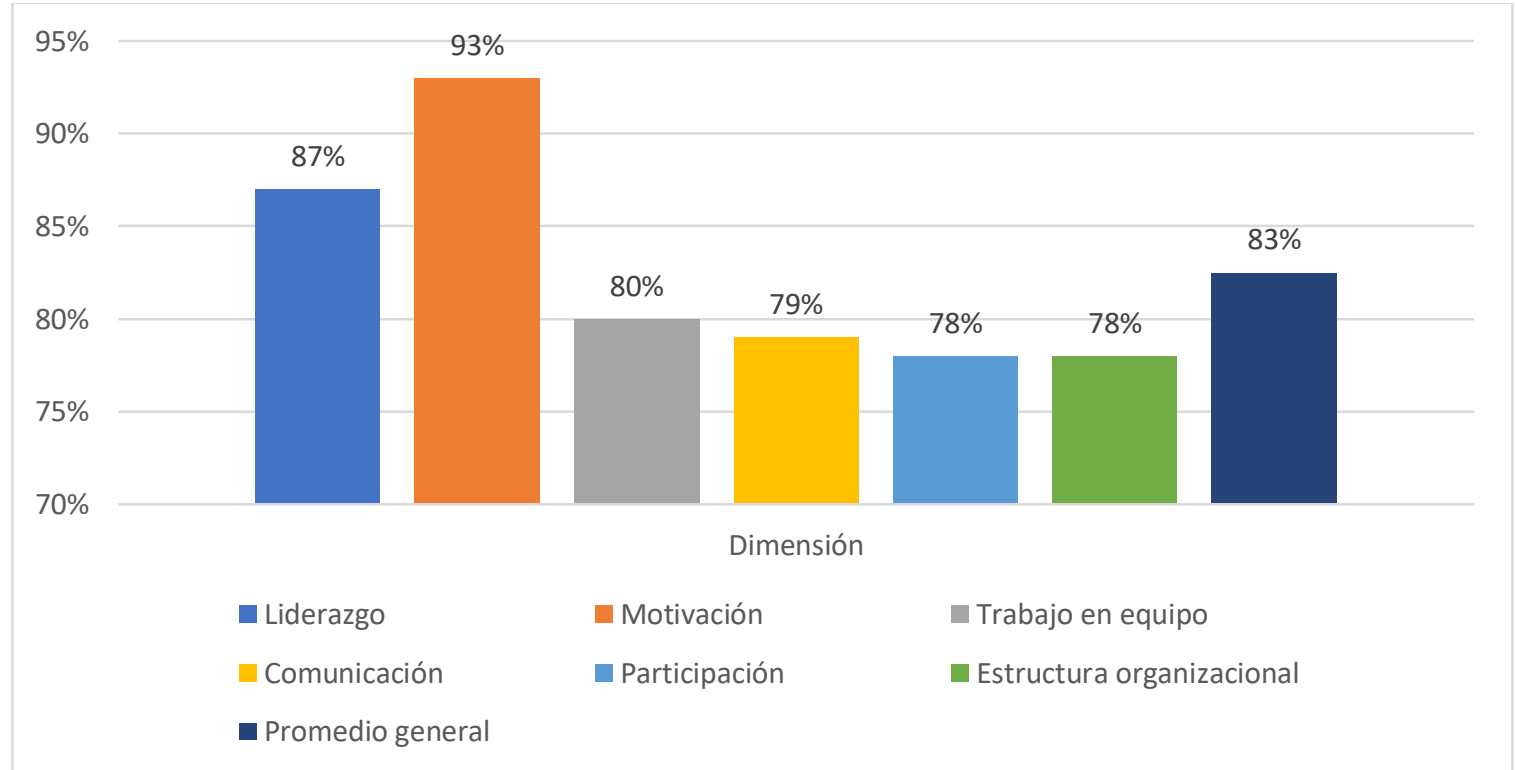

Gráfico 1. Resultados de la variable de Competencias Organizacionales 
Comprobando los resultados generalizados de cada dimensión de la variable de Competencias Organizacionales, es posible evidenciar que el ítem con mayor valoración es la motivación, con un $93 \%$ de satisfacción dentro de la muestra de estudio; este es el único indicador con un nivel 'satisfactorio', de lo cual discrepan los aspectos de participación y estructura organizacional, ambas con una valoración de $78 \%$, y a su vez consideradas regulares.

En este sentido, partiendo de la media ponderada de todos los parámetros evaluados de la variable, se halla un nivel de satisfacción del 83\%, cualificado como aceptable; esto determina que, si bien las competencias organizacionales no se hallan en un comportamiento ideal, es perfectamente mejorable si se aplican las estrategias intervencionistas adecuadas a cada falencia detectada.

\section{Desempeño Laboral}

Tabla 2

Resultados de la variable de Desempeño Laboral

\begin{tabular}{ccccc}
\hline $\mathrm{N}^{\circ}$ & Dimensión & $\begin{array}{c}\text { Media } \\
\text { ponderada }\end{array}$ & Porcentaje & Evaluación \\
\hline $\mathbf{1}$ & Puesto de Trabajo & 4,19 & $84 \%$ & Aceptable \\
$\mathbf{2}$ & Línea de autoridad & 4,24 & $85 \%$ & Aceptable \\
$\mathbf{3}$ & Ambiente de trabajo e integración & 4,06 & $81 \%$ & Aceptable \\
$\mathbf{4}$ & Comunicación y coordinación & 3,83 & $77 \%$ & Regular \\
$\mathbf{5}$ & Condiciones ambientales, infraestructura & 4,14 & $83 \%$ & Aceptable \\
$\mathbf{6}$ & y recursos & 4,20 & $84 \%$ & Aceptable \\
$\mathbf{7}$ & Implicación en la mejora & 4,42 & $88 \%$ & Aceptable \\
Total & Motivación y reconocimiento & 4,15 & $83 \%$ & Aceptable \\
\hline
\end{tabular}




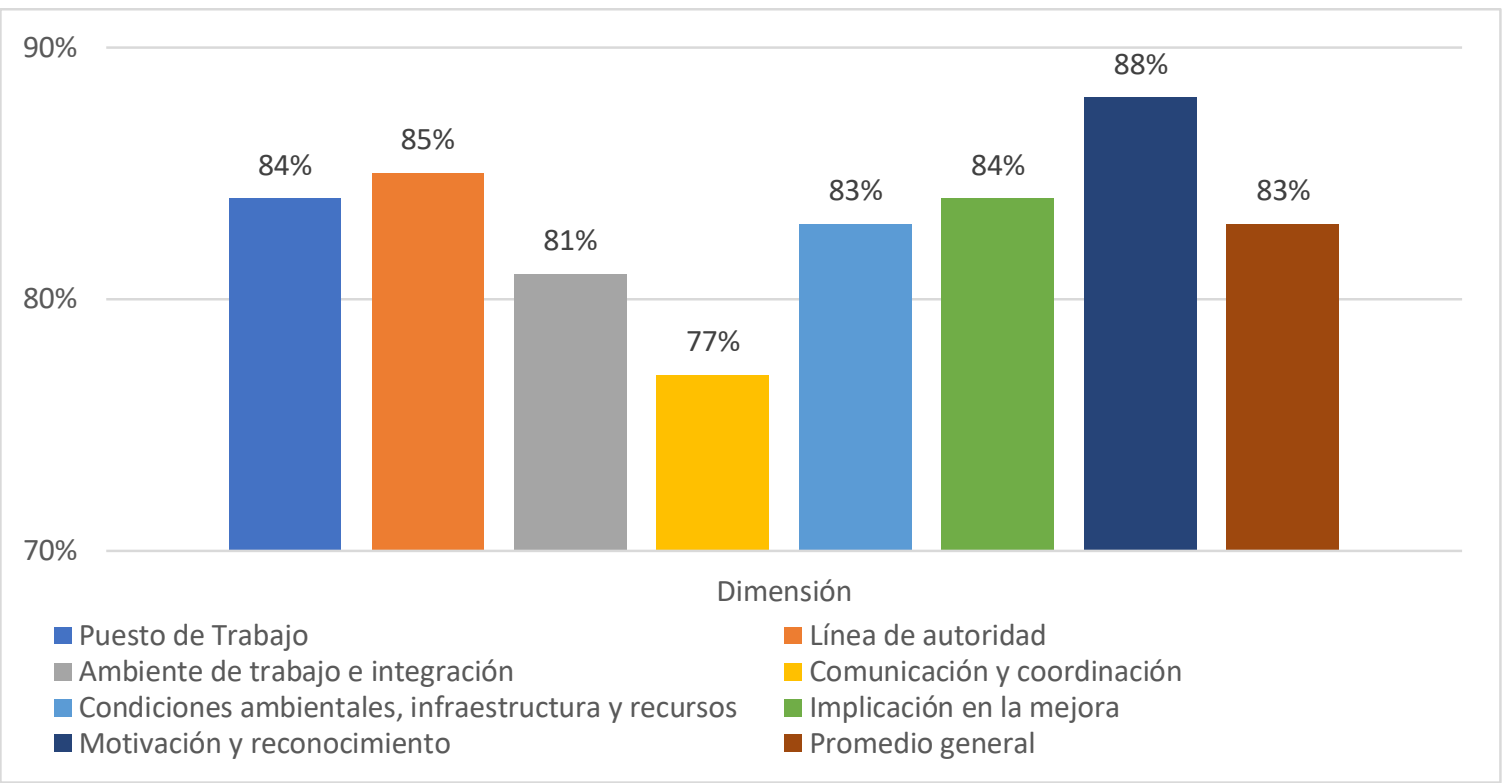

Gráfico 2. Resultados de la variable de Desempeño Laboral

En otro orden de ideas, dentro de la segunda variable de “desempeño laboral," es posible evidenciar que el indicador con mayor ponderación corresponde a la motivación y reconocimiento, con un $88 \%$ que lo sitúa como aceptable, frente a la comunicación y coordinación, que discrepa de este aspecto con un 77\%, como la de menor valoración en este sentido.

Asimismo, se halla una valoración generalizada de un $83 \%$ dentro de esta variable, lo cual es considerado aceptable, al igual que dentro de las competencias organizacionales, ello pese a que dentro de este ítem no exista ningún indicador con valoración satisfactoria, aunque exista un mayor equilibrio entre múltiples aspectos de análisis.

Correlación entre las dos variables

Tabla 3

Resultados de la correlación entre ambas variables

\begin{tabular}{ccccc}
\hline $\mathrm{N}^{\circ}$ & Variable & $\begin{array}{c}\text { Media } \\
\text { ponderada }\end{array}$ & Porcentaje & Evaluación \\
\hline $\mathbf{1}$ & Competencias organizacionales & 4,13 & $85 \%$ & Aceptable \\
$\mathbf{2}$ & Desempeño laboral & 4,15 & $83 \%$ & Aceptable \\
Total & Promedio general & 4,14 & $84 \%$ & Aceptable \\
\hline
\end{tabular}




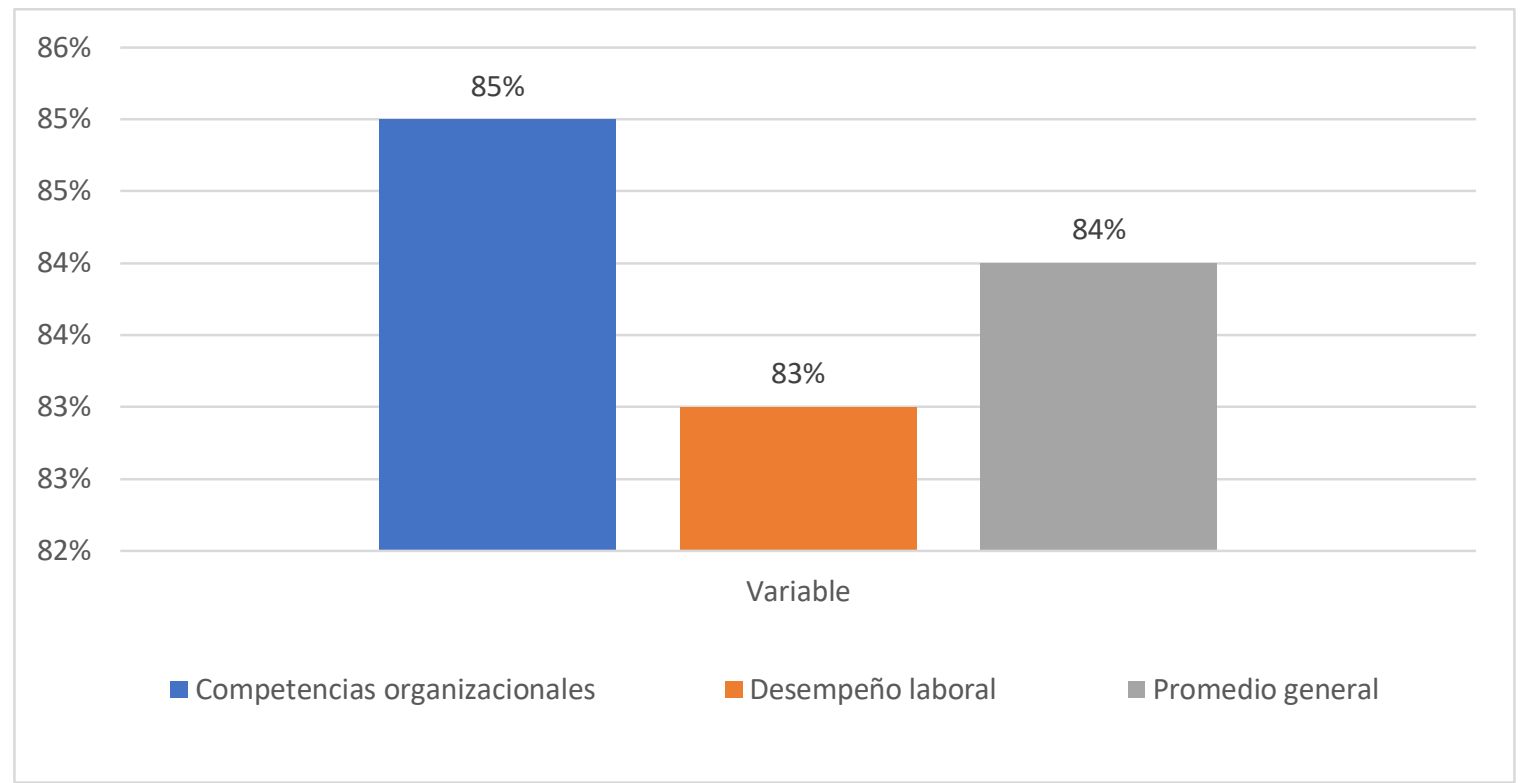

Gráfico 3. Resultados de la correlación entre ambas variables

Ahora bien, cotejando la correlación entre ambas variables, se determina que las competencias organizacionales presentan un $85 \%$, frente al $83 \%$ del desempeño laboral, con lo cual es posible constatar un promedio general de $84 \%$; esto se considera aceptable dentro de la escala de valoración preconcebida. En este sentido, se determina que, si bien ambos aspectos se consideran adecuados, existen derivaciones que sí podrían ser potenciadas a partir de la consecución de estrategias intervencionistas que potencien el desempeño laboral y las competencias organizacionales como elementos ampliamente interrelacionados e interdependientes.

Por ello, a fin de determinar la relación existente entre las variables de desempeño laboral y las dimensiones de competencias organizaciones, fue determinado el valor del Chi Cuadrado de Pearson, según corresponda. Inicialmente, se plantean las hipótesis nula y alternativa con el objetivo de determinar el valor de la significancia de la prueba estadística (p-valor) y compararlo con el valor teórico, generalmente aceptado como 0.05.

Así, tal como se puede apreciar para todos los casos analizados, se determinó que existe asociación estadísticamente significativa entre las dimensiones de competencias organizaciones (liderazgo, motivación, trabajo en equipo, comunicación, participación, estructura organizacional) y el desempeño laboral de los integrantes de la iglesia. 


\section{Conclusiones}

La presente investigación pretendió establecer una correlación entre las variables de desempeño laboral y competencias organizacionales en una muestra de estudio compuesta por pastores de la IASD de la ciudad de Campinas, perteneciente a Sao Paulo, a partir de una serie de parámetros metodológicos de carácter descriptivo, no experimental y correlacional.

En este orden de ideas, fue posible estructurar un cuestionario como instrumento de recolección de información, dividido en dos variables de acción, las cuales comprendían un conjunto de indicadores evaluativos conformados por una serie de interrogantes que permitieran conocer su comportamiento generalizado. Con ello se establecerían parámetros de mejoramiento para los factores de comportamiento que comprenden a toda actividad colectiva o de carácter empresarial.

Así, verificando aspectos de valoración como el 93\% hallado en el indicador de motivación-único aspecto valorado como satisfactorio-determina que existen aspectos potenciados dentro de este entorno de convivencia, ello pese a la existencia del $77 \%$ presente en la comunicación y coordinación (considerado regular y de menor valoración en la encuesta), más en consideración de la importancia de la correcta construcción de redes de relacionamiento humano, fundamentadas en la cooperación y en el logro de objetivos conjuntamente constituidos.

No obstante, pese a no hallarse ningún aspecto de carácter deficiente, es necesario establecer políticas de mejoramiento de procesos dentro de esta comunidad dependiente de una identidad tan mundialmente reconocida como lo es la IASD, fundamentada en el liderazgo, el servicio y otros factores que fundamentan a una institución de carácter religioso con tal movimiento de masas como esta.

Por lo tanto, en materia de recomendaciones, se establece la importancia en el establecimiento de políticas y acciones estratégicas en pro al mejoramiento del comportamiento en el desempeño laboral de sus empleados; aquí también se identifican diferenciaciones entre los roles de cada individuo o departamento, a su vez que garantiza un clima laboral adecuado que propicie al mejoramiento del rendimiento individual que surge del reconocimiento de las acciones de los empleados y el fomento a la autorrealización y a su identificación como parte importante de la institución global IASD. 
Asimismo, es necesario demarcar las implicaciones de las competencias laborales de esta empresa, a partir de la potenciación del liderazgo como una cualidad presente en la totalidad de los sujetos de cambio, en consonancia con su implicación como emisores de continuos mensajes y pautas de comportamiento hacia sus receptores. Por ello, es necesario potencializar las aptitudes y destrezas dentro de su campo de acción, con miras a generar ambientes adecuados a los objetivos de esta institución.

Así, de manera generalizada, es importante establecer la importancia que tiene el talento humano de una organización, la que debe ser administrada de manera eficiente y adecuada, buscando satisfacer sus necesidades y aprovechar la experiencia, habilidades y destrezas que han conseguido los líderes locales para la consecución de sus objetivos.

Ahora bien, al establecer las relaciones existentes entre las variables de estudio, se determina que las competencias laborales están estrechamente ligadas al desempeño laboral, partiendo del principio que las cualificaciones que tenga un individuo, en consonancia al ambiente que se establezca en su contexto de acción, intervienen de manera directa en los resultados obtenidos, donde a su vez se contempla la motivación como un valor añadido a este proceso.

Por lo tanto, en respuesta a los objetivos, se establece que, en la IASD, se presenta una estrecha relación entre las dos variables de estudio, donde aspectos como el liderazgo, la motivación, el trabajo en equipo, la estructura organizacional y la participación inciden de manera directa en el direccionamiento de una empresa como esta, reconocida a nivel mundial por sus funciones, por lo que la correcta observación y una adecuación de estos indicadores a los estándares ideales, permitirá un desarrollo independiente de los integrantes del talento humano y a su vez de la organización como un todo.

\section{Referencias}

Aguirre, J. (2000). Dirección y gestión de personal. Madrid: Ediciones pirámide.

Aristizábal, C., Ospina, B., \& Ramírez, M. (2003). La escala de Likert en la valoración de los conocimientos y las actitudes de los profesionales de. Investigación y Educación en Enfermería, XXIII(1), 14-29. Obtenido de http://www.redalyc.org/pdf/1052/105215401002.pdf 
Baptista, M., Fernández, C., \& Sampieri, R. (2014). Metodología de la investigación. Mexico: Mc Graw Hill - Education. Obtenido de https://docs.google.com/viewer?a=v\&pid=sites\&srcid=ZGVmYXVsdGRvbWFpbn xjb250YWR1cmlhcHVibGljYTk5MDUxMHxneDo0NmMxMTY0NzkxNzliZmY $\mathrm{W}$

Barrios, E., \& Fong, M. (2002). Diseño Curricular Basado en Competencias. Santiago de Chile, Chile: INTECAP (Instituto Técnico de Capacitación y Producción). Obtenido de

http://eoepsabi.educa.aragon.es/descargas/H_Recursos/h_1_Psicol_Educacion/h_1.4 .Eval_desemp_docente/1.3.Anteced_eval_desemp.pdf

Bohlander, G., \& Sherman, A. (2011). Administración de los Recursos Humanos 3a. ed. México: Prentice-Hall Hispanoamericana.

Camejo, A. (2008). El modelo de gestión por competencias y la evaluación del desempeño en la gerencia de los recursos humanos. Entelequia. Revista Interdisciplinar,(8), $97-$ 116. Obtenido de http://www.avatarconsultores.com.mx/wpcontent/uploads/2013/07/Evaluaci\%C3\%B3n-por-competencias.pdf

Dávila, J. (2013). Capacidades organizacionales: dinámicas por naturaleza. Cuadernos de Administración, 26(47), 11-33. Obtenido de http://www.redalyc.org/pdf/205/20531182002.pdf

Ivancevich, J. (2005). Administración de Recursos Humanos. McGraw Hill: México.

Merten, L. (2000). Competencia laboral: sistemas, surgimiento y modelos. CINTERFOR/OIT: Montevideo.

Monje, C. (2011). Metodología de la Investigación Cuantitativa Y Cualitativa. Guía Didáctica. Neiva - Colombia: Universidad Surcolombiana. Obtenido de https://carmonje.wikispaces.com/file/view/Monje+Carlos+Arturo++Gu\%C3\%ADa+did\%C3\%A1ctica+Metodolog\%C3\%ADa+de+la+investigaci\%C3 $\%$ B3n.pdf

Tinoco, O. (2008). Una aplicación de la prueba chi cuadrado con SPSS. Industrial Data, 11(1), 73-77. Obtenido de http://www.redalyc.org/pdf/816/81611211011.pdf

Universidad de Salamanca. (2004). Cuestionario de desempeño laboral del personal administrativo y servicios. Salamanca: UDS. 
